\title{
ВЛИЯНИЕ КВАДРАТИЧНОГО ВНУТРИЗОННОГО ВИБРОННОГО ВЗАИМОДЕЙСТВИЯ НА ХАРАКТЕРИСТИКИ СЕГНЕТОЭЛЕКТРИЧЕСКИХ ФАЗОВЫХ ПЕРЕХОДОВ ТИПА СМЕЩЕНИЯ
}

\author{
(Представил В. Хижняков)
}

В рамках вибронной теории рассчитана завнсимость спонтанного искажения решетки, частот мягких мод, температуры Кюри-Вейсса и других характеристик сегнетоэлектрических фазовых переходов типа смещения от квадратичного внутризонного вибронного взаимодействия (KBB). При определенном соотношении между параметрами теории в узкощельном сегнетоэлектрыке-полупроводкике возможен фазовый переход первого рода.

1. Согласно вибронной теории сегнетоэлекпрические фазовые переходы (СФП) типа смещения индуцируются межзонным электрон-фононным взаимодействием (см., напр., $\left[{ }^{1,2}\right]$ ). Константы линейного внутризопного вибронного взаимодействия для сегнетоактивных $\mathbf{q}=0$ (q - волновой вектор фононов) колебаний из соображений симметрии равны нулю. Учет этого взаимодействия становится существенным при расчете зависимости частоты мягкой моды от $\mathbf{q}(\mathbf{q} \neq 0)$. Линейное внутризонное вибронное взаимодействие при $q \neq 0$ может также индуцировать фазовые переходы, но не собственно сегнетоэлектрического типа (несоразмерные, пайерлсовские и антисепнетоэлектрические) $\left[{ }^{2}\right]$. Константы КВВ отличны от нуля уже при $\mathrm{q}=0$ и можно ожидать непосредственного влияния КВВ на СФП $\left[{ }^{3,4}\right]$. КВВ привлекалось * также для объяснения аномального электросопротивления $\mathrm{BaTiO}_{3}$ в кубической и тетрагональной фазах $\left[{ }^{8}\right]$.

В данной работе нами проведен детальный расчет характеристик СФП с учетом внутризонного КВВ, причем рассматривается существенно более общая модель в сравнении с моделью из [ $\left.{ }^{4}\right]$.

2. Запишем гамильтониан электрон-фононной системы в виде

$$
\begin{aligned}
& H=-\sum_{\sigma, \mathrm{k}} \varepsilon_{\sigma}(\mathrm{k}) a_{\sigma \mathrm{k}}^{+} a_{\sigma \mathrm{k}}+\frac{1}{2}\left(M^{-1} P_{0}^{2}+M \omega_{0}^{2} y_{0}^{2}\right)+ \\
& +N_{0}^{-1 / 2} \sum_{\sigma, \sigma^{\prime}} \sum_{\mathrm{k}} a_{\sigma \mathrm{k}}^{+} a_{\sigma^{\prime} \mathrm{k}}\left(V_{\sigma \sigma^{\prime}}(0) y_{0}+N_{0}^{-1 / 2} K_{\sigma \sigma^{\prime}}(0) y_{0}^{2}\right) .
\end{aligned}
$$

Здесь $\varepsilon_{\sigma}(\mathbf{k})$ - исходные спектры валентной зоны $(\sigma=1)$ и зоны проводимости $(\sigma=2), a^{+}, a$ - операторы рождения и уничтожения электронов, $y_{0}-$ нормальные координаты и $P_{0}-$ сопряженные им импульсы сегнетоактивного оптического колебания, $M$ - соответствующая приве-

* В [ ${ }^{5,6}$ ] изучались эффекты квадратичного вибронного взанмодействия сегпетоактивной мягкой моды с системой ян-теллеровских прнмесных центров. Известно также, что КВВ может существенно влиять на оптические свойства примесных центров (см., напр., $\left.\left[{ }^{7}\right]\right)$. 
денная масса, $N_{0}$ - число элементарных ячеек в кристалле. Последний член в (1) описывает электрон-фононные взаимодействия. Для случяя электронных зон противоположной четности $V_{\sigma \sigma^{\prime}}(0)=\left(1-\delta_{\sigma \sigma^{\prime}}\right) V$ и $K_{\sigma \sigma^{\prime}}(0)=\delta_{\sigma \sigma^{\prime}} K_{\sigma}$, где $V$ и $K_{\sigma}(\sigma=1,2)$ - соответственно жонстанты линейного межзонного и квадратичного внутризонного взаимодействий для сегнетоактивной моды.

Для нахождения электронного спектра на основе гамильтониана (1) составлялась цепочка уравнений для двухвременных функций Грина $\ll a_{\sigma \mathrm{k}}(t) ; a_{\sigma^{\prime} \mathrm{k}^{\prime}}^{+}(0) \gg$. В приближении среднего поля для перенормированных вибронными взаимодействиями эиергетических спектров валентной зоны и зоны проводимости получается

$$
E_{1,2}(\mathbf{k})=\frac{1}{2}\left\{\varepsilon_{1}(\mathbf{k})+\varepsilon_{2}(\mathrm{k})+N_{0}^{-1}\left(K_{1}+K_{2}\right) y_{0}^{2} \mp E_{g}\left(\mathrm{k}, y_{0}\right)\right\},
$$

где

$$
E_{\mathbf{g}}\left(\mathbf{k}, y_{0}\right)=\left\{\left[\varepsilon_{2}(\mathbf{k})-\varepsilon_{1}(\mathbf{k})+N_{0}^{-1}\left(K_{2}-K_{1}\right) y_{0}^{2}\right]^{2}+\frac{4 V^{2}}{N_{0}} y_{0}^{2}\right\}^{1 / 2}
$$

С учетом (1)-(3), аналогично $\left[{ }^{2}\right]$, можно записать свободную энергию системы $F\left(T, y_{0}\right)$ в виде

$$
F\left(T, y_{0}\right)=N \mu-k_{B} T \sum_{v, \mathbf{k}} \ln \left[1+\exp \frac{\mu-E_{v}\left(\mathbf{k}, y_{0}\right)}{k_{B} T}\right]+\frac{M \omega_{0}^{2}}{2} y_{0}^{2},
$$

причем

$$
\sum_{v, \mathrm{k}} f_{v}\left(\mathbf{k}, y_{0}\right)=N
$$

В (5) числа заполнения электронных зон равны

$$
f_{v}\left(\mathbf{k}, y_{0}\right)=\left[1+\exp \frac{E_{v}\left(\mathbf{k}, y_{0}\right)-\mu}{k_{B} T}\right]^{-1},
$$

где $N$ - число электронов в системе и $\mu-$ химический потенциал.

Из условия $\frac{\partial F}{\partial y_{0} \mid y_{00}}=0$ с учетом (6) получена следующая система связанных уравнений для нахождения параметра порядка $y_{00} / \sqrt{N_{0}}$ и химического потенциала $\mu\left(y_{00}\right)$ (при этом должно выполняться условие устойчивости сегнетофазы $\left.\frac{\partial^{2} F}{\partial y_{0}^{2}} \mid y_{00}>0\right)$

$$
\left\{\begin{array}{l}
\frac{1}{2 N_{0}} \sum_{\mathrm{k}}\left[\left(2 V^{2}+\left(K_{2}-K_{1}\right)\right)\left[\varepsilon_{2}(\mathbf{k})-\varepsilon_{1}(\mathbf{k})+\left(K_{2}-K_{1}\right) \frac{y_{0 \theta}^{2}}{N_{0}}\right] E_{g}^{-1}\left(\mathbf{k}, y_{00}\right) X\right. \\
\left.X\left\{\operatorname{th} \frac{E_{g}\left(\mathbf{k}, y_{00}\right)+u\left(\mathbf{k}, y_{00}\right)}{4 k_{B} T}+\operatorname{th} \frac{E_{g}\left(\mathbf{k}, y_{00}\right)-u\left(\mathbf{k}, y_{00}\right)}{4 k_{B} T}\right\}\right]=M \omega_{0}^{2}+\bar{K}_{1}+\bar{K}_{2}, \\
\sum_{v, \mathbf{k}} f_{v}\left(\mathbf{k}, y_{00}\right)=N,
\end{array}\right.
$$

где

$u\left(\mathbf{k}, y_{00}\right)=2 \mu-\left[\varepsilon_{1}(\mathbf{k})+\varepsilon_{2}(\mathbf{k})+N_{0}^{-1}\left(K_{1}+K_{2}\right) y_{00}^{2}\right], \quad \bar{K}_{1,2}=K_{1,2} \frac{N}{N_{0}}$.

Для случая предельно узких зон $\mu=\frac{1}{2}\left(\Delta+\left(K_{1}+K_{2}\right) N_{0}^{-1} y_{00}^{2}\right)$, где 
$\Delta$ - затравочная эффективная щель. Тогда (7) принимает вид $\left.\overline{\left(M \omega_{0}^{2}\right.}=M \omega_{0}^{2} N_{0} / N\right)$

$$
\overline{M \omega_{0}^{2}}(1+\beta)=\frac{2 V^{2}\left(1+\alpha+\alpha^{2} \frac{2 V^{2} y_{00}^{2}}{N_{0} \Delta^{2}}\right)}{E_{g}\left(0, y_{00}\right)} \text { th } \frac{E_{g}\left(0, y_{00}\right)}{4 k_{B} T} \text {. }
$$

Здесь введены безразмерные параметры

$$
\alpha=\frac{\left(K_{2}-K_{1}\right) \Delta}{2 V^{2}} \text { и } \beta=\frac{K_{1}+K_{2}}{\overline{M \omega_{0}^{2}}} .
$$

Разлагая $F\left(T, y_{0}\right)$ в ряд по $y_{0}$, около равновесных значений $y_{00}$ получаем квадраты частот мягкой моды для высокосимметричной и низкосимметричной фаз

$$
\begin{gathered}
\frac{\partial^{2} F}{\partial y_{0}^{2}} \mid y_{0}=0 \equiv M \Omega_{\text {вс. }}^{2}(T)=\frac{N}{N_{0}}\left\{\overline{M \omega_{0}^{2}}(1+\beta)-\frac{2 V^{2}(1+\alpha)}{\Delta} \text { th } \frac{\Delta}{4 k_{B} T}\right\}, \\
\left.\frac{\partial^{2} F}{\partial y_{0}^{2}}\right|_{y_{0}}=y_{00} \equiv M \Omega_{\text {нс. }}^{2}(T)=\frac{N}{N_{0}^{2}} \frac{8 V^{4} y_{00}^{2}}{E_{g}^{2}\left(0, y_{00}\right)}\left\{\frac{1+2 \alpha}{E_{g}\left(0, y_{00}\right)} \text { th } \frac{E_{g}\left(0, y_{00}\right)}{4 k_{B} T}-\right. \\
\left.-\frac{\left(1+\alpha+\alpha^{2} \frac{2 V^{2} y_{00}^{2}}{N_{0} \Delta^{2}}\right)^{2}}{4 k_{B} T} \operatorname{ch}^{-2} \frac{E_{g}\left(0, y_{00}\right)}{4 k_{B} T}\right\}
\end{gathered}
$$
если

Из (9), (11) и (12) следует, что при $T=0$ реализуется сегнетофаза,

$$
\beta>-1 \text { и } \frac{1+\beta}{\tau_{0}}>\alpha>\left\{\begin{array}{l}
-\frac{1+\beta}{\tau_{0}}, \text { при } \beta<\frac{1}{2} \tau_{0}-1 \\
\frac{1+\beta}{\tau_{0}}-1 ; \text { при } \beta \geqslant \frac{1}{2} \tau_{0}-1,
\end{array}\right.
$$

где $\tau_{0}=2 V^{2} /\left(\overline{M \omega_{0}^{2}} \Delta\right)$.

В отсутствие межзонного вибронного взаимодействия $(V \equiv 0)$ внутризонное КВВ может вызвать сегнетоэлектрическую динамическую неустойчивость парафазы, если ${\overline{M \omega_{0}}}_{2}<-2 K_{1}$. Однако КВВ при этом не поставляет необходимую для стабилизации сегнетофазы ангармоничность *.

Температурная зависимость спонтанного искажения решетки $y_{00}(T)$ для модели узкощельного сегнетоэлектрика-полупроводника, найденная из уравнения (9) (рис. 1), показывает, что с ростом $\alpha y_{00}$ значительно увеличивается. При этом в районе СФП температурная зависимость параметра порядка становится более резкой и при превышении порогового значения $\alpha_{k} \approx 0,94$ (при выбранном значении параметра $\beta=0,2$ ) СФП второго рода превращается в переход первого рода. Это имеет место, когда энергии линейного и квадратичного электрон-фононных взаимодействий будут одного порядка, т. е. $K y_{0}^{2} \sim V y_{0} N_{0}^{1 / 2}$.

Области значений параметров $\alpha$ и $\beta$, при которых сегнетофаза устой-

* Такой СФП может быть стабилизирован, например, фононным ангармонизмом (cм. $\left.\left\lfloor{ }^{4}\right\rfloor\right)$. 


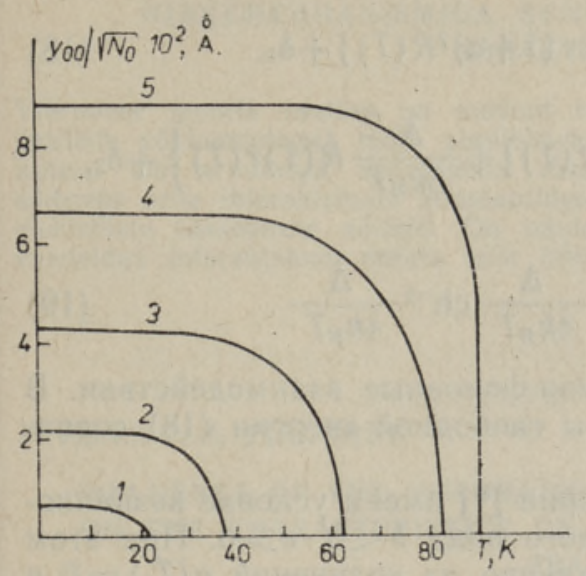

Рис. 1.

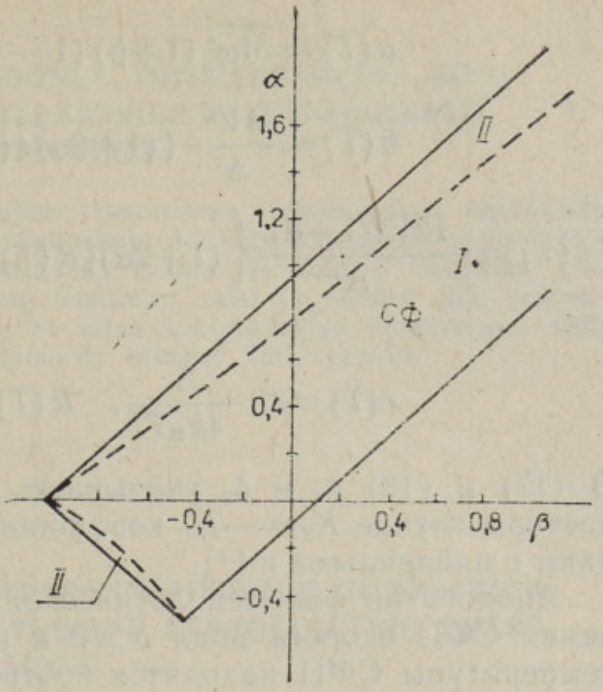

Рис. 2.

Рис. 1. Зависимость спонтанного искажения решетки от температуры при различных значениях $\alpha: 1-\alpha=0,1 ; 2-\alpha=0,2 ; 3-\alpha=0,5 ; 4-\alpha=0,8 ; 5-\alpha=0,94$. Использованные значения параметров теории: $V=0,2$ эВ $/ \AA, \Delta=0,02$ эВ, $\overline{M \omega_{0}^{2}}=$ $=3,62$ эВ $/ \AA^{2}$.

Рис. 2. Область значений параметров $\alpha$ и $\beta$, при которых сегнетофаза (СФ) устойчива; $\tau_{0}=1,1$. I - область реализации СФП второго рода, II - СФП первого рода.

чива, показаны на рис. 2 сплошными линиями. Кривая $\alpha_{k}=\alpha_{k}\left(\beta_{k}\right)$, разделяющая СФП второго и первого рода, выделена пунктиром.

Температура Кюри-Вейсса, определяемая условием $M \Omega_{\text {вс. }}{ }^{2}\left(T_{c}\right)=0$, равна

$$
k_{B} T_{c}=\frac{\Delta}{4}\{\operatorname{Arcth} \tau\}^{-1},
$$

где

$$
\tau=\frac{1+\alpha}{1+\beta} \tau_{0}=\frac{2 V^{2}+\Delta\left(K_{2}-K_{1}\right)}{\left(\overline{M \omega_{0}^{2}}+K_{1}+K_{2}\right) \Delta} .
$$

Согласно (14), электрон-фононные взаимодействия вызывают динамическую сегнетоэлектрическую неустойчивость $\left(T_{c}>0\right)$, если

$$
\tau>1 \text {. }
$$

Критерий (16) аналогичен необходимому условию $\tau_{0}>1$ в теории с учетом только линейного межзонного вибронного взаимодействия [ $\left.{ }^{1}\right]$.

При выполнении соотношений (13) вибронное взаимодействие само по себе способно стабилизировать сегнетофазу. Из (16) следует, что $\mathrm{KBB}$ при $K_{1}<0$ действует в сторону возникновения динамической неустойчивости, а при $K_{1}>0$ расширяет область стабильности парафазы.

3. Для более подробного изучения механизма СФП первого рода разложим свободную энергию в ряд по степеням $y_{0}$, добавляя сразу фононную ангармоничность. Тогда имеем

где

$$
F\left(T, y_{0}\right)=\frac{N a(T)}{2 N_{0}} y_{0}^{2}+\frac{N b(T)}{4 N_{0}^{2}} y_{0}^{4}+\frac{N c(T)}{6 N_{0}^{3}} y_{0}^{6}+\ldots,
$$




$$
\begin{gathered}
a(T)=\overline{M \omega_{0}^{2}}(1+\beta)(1-\tau r(T)), \\
b(T)=\frac{4 V^{4}}{\Delta^{3}}\left\{(1+2 \alpha) r(T)-(1+\alpha)^{2} R(T)\right\}+\delta_{1}, \\
c(T)=\frac{12 V^{6}(1+\alpha)}{\Delta^{5}}\left\{(1+2 \alpha)[R(T)-r(T)]+\frac{\Delta}{6 k_{B} T} R(T) r(T)\right\}+\delta_{2},
\end{gathered}
$$

где

$$
r(T)=\operatorname{th} \frac{\Delta}{4 k_{B} T}, \quad R(T)=\frac{\Delta}{4 k_{B} T} \operatorname{ch}^{-2} \frac{\Delta}{4 k_{B} T} .
$$

В (17) и (18) $\delta_{1}$ и $\delta_{2}$ учитывают фонон-фононные взаимодействия. В частном случае $K_{2}=-K_{1}$ коэффициенты свободной энергии (18) совпадают с найденными в $\left[{ }^{4}\right]$.

Аналогично феноменологической теории $\left[{ }^{9}\right]$ имеем условие возникновения СФП второго рода $b>0$ и первого рода $b<0, c>0$. При этом температуры СФП находятся соответственно из уравнений $a\left(T_{c}\right)=0$ и $3 b^{2}\left(T_{0}\right)=16 a\left(T_{0}\right) c\left(T_{0}\right)$. Анализ показывает, что при $\delta_{1}=\delta_{2}=0$ возможно превращение СФП второго рода в переход первого рода. (Заметим, что учет фонон-фононных взаимодействий может также привести к реализации СФП первого рода.) Физическая причина такого превращения состоит в том, что КВВ приводит к изменению чисел заполнения электронных зон и, как следствие, при некоторых $\alpha, \beta$ коэффициент $b(T)$ с ростом температуры становится отрицательным, а $c(T)$ - положительным еще ниже точки перехода.

В заключение отметим, что предложенный выше механизм СФП первого рода может реализоваться только в случае сегнетоэлектриковполупроводников с узкой запрещенной зоной, т. е. когда электрон-фононный ангармонизм играет существенную роль. Похожий механизм СФП первого рода можно предложить для объяснения смены рода перехода под влиянием примесных носителей.

Авторы выражают глубокую благодарность Н. Кристофелю за обсуждение результатов работы и ценные замечания.

\section{ЛИТЕРАТУРА}

1. Кристофель Н. Н., Консин П. Н. Успехи физ. наук, 120, вып, 3, 507-510 (1976).

2. Konsin, $P$. Phys. status solidi (b), 76, № 2, 487-496 (1976); 86, № 1, 57-66 (1978)

3. Konsin, P. Ferroelectrics, 45, 45-50 (1982).

4. Вихнин В. С., Орлов О. Л. Физ. твердого тела, 24, вып. 6, 1665-1668 (1982).

5. Кристофель Н. Н. Физ. твердого тела, 23, вып. 11, 3267-3272 (1981).

6. Вихнин В. С. Физ. твердого тела, 23, вып. 8, 2370-2375 (1981).

7. Кристофель Н. Н. Теория примесных центров малых радиусов в ионных кристаллах. M., «Наука», 1974.

8. Imaseki, T., Kinase, W. Phys, Rev., B27, 1228-1232 (1983).

9. Смоленский $\Gamma$. А., Боков В. А., Исупов В. А., Крайник Н. Н., Пасынков Р. E., Щур М. С. Сегнетоэлектрики и антнсегнетоэлектрнки. Л., «Наука», 1971.

Ннститут физики

Академии наук Эстонской ССР
Поступила в редакцию $31 /$ X 1983 


\section{RUUDULISE TSOONISISESE VIBROONSE INTERAKTSIOONI MOJU NIHKEMEHHANISMIGA SENJETTELEKTRILISTE FAASISIIRETE KARAKTERISTIKUTELE}

Vibroonse teooria raames on uuritud ruudulise tsoonisisese interaktsiooni (aktiivsete optiliste võnkumistega) mõju pooljuht-senjettelektrikute senjettelektrilistele karakteristikutele. On arvutatud spontaanse võremoonutuse, pehme võnkumise sageduse jm. sõltuvus selle interaktsiooni konstantidest ning käsitletud teist ja esimest liiki senjettelektriliste faasisiirete näiteid. On näidatud, et kitsa keelutsooniga süsteemides võib vaadeldav interaktsioon muuta teist liiki faasisiirde esimest liiki siirdeks.

\section{P. RONSIN, A. PISHCHEV}

\section{INFLUENCE OF THE QUADRATIC INTRABAND VIBRONIC INTERACTION ON THE CHARACTERISTICS OF DISPLACIVE FERROELECTRIC PHASE TRANSITIONS}

In the iramework of the vibronic theory the influence of the quadratic intraband vibronic interaction (QVI) with active optic vibrations on the ferroelectric characteristics of ferroelectric semiconductors, is investigated. The dependence of the spontaneous lattice distortion of the soft mode frequency, on the constants of QVI, is calculated. Examples of the first-and-second-kind transitions are considered. It is shown that in the case of a narrow-gap system the QVI may change the second-kind transition into a first-kind one. 